
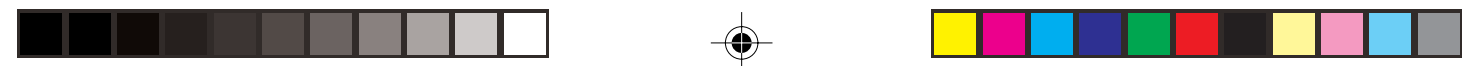

\title{
REGISTROS IMPORTANTES DE MAMÍFEROS PARA CAMPECHE, MÉXICO
}

\author{
GRISELDA ESCALONA-SEGURA ${ }^{1}$, JORGE A. VARGAS-CONTRERAS ${ }^{2}$ \\ Y LUDIVINA INTERIÁN-SOSA ${ }^{1}$
}

${ }^{1}$ El Colegio de la Frontera Sur (ECOSUR), Unidad Campeche, Calle 10 No. 264, Colonia Centro, 24000 Campeche, Campeche, México,e-mail: gescalon@camp.ecosur.mx ${ }^{2}$ Instituto de Ecología, Universidad Nacional Autónoma de México, e-mail: jalbino@miranda.ecologia.unam,mx

Ap. Postal 70-275, México, D. F.

Palabras claves: Chiroptera, Carnivora, Cueva, Calakmul, Campeche.

El estado de Campeche presenta extensiones considerables de selva en buen estado de conservación y factibles para realizar diversos estudios sobre la distribución de especies, ecológicos y de conservación de los vertebrados y de la flora. Recientemente, esta zona ha sido de interés para reportar información relevante, entre la que destaca la referente a nuevos registros de mamíferos para la Peninsula de Yucatán tales como Micronycteris brachyotis, Vampyrum spectrum y Bauerus dubiaquercus (HernándezHuerta et al., 2000; Vargas-Contreras et al., en prensa; Vargas Contreras et al., en revisión).

En Campeche se han reportado 88 especies de mamíferos, de las cuales 38 son quirópteros y 13 son carnívoros (Genoways y Jones, 1975; Hall, 1981; HernándezHuerta et al., 2000; Jones et al., 1973, 1974a, 1974b; Medellín et al., 1998; Sánchez y Romero, 1995; Vargas-Contreras et al., en prensa; Vargas-Contreras et al., en revisión). Sin embargo, aunque se tienen listas sobre los mamíferos del Estado, aún existen áreas inexploradas.

El presente trabajo tiene el propósito de documentar registros notables de mamíferos en el estado de Campeche, particularmente de especies de los órdenes Chiroptera y Carnivora durante los años de 2000 al 2002 en el centro y sur de Campeche.

En este escrito se incluyen sitios localizados en el centro - oeste y sur de Campeche, los cuales se caracterizan a continuación: a) en el centro - oeste de Campeche se encuentran diferentes tipos de vegetación como la vegetación hidrófita, vegetación modificada, selva baja y mediana (Flores y Espejel 1994; INEGI, 2001). Hay un clima cálido subhúmedo, con lluvias en verano que alcanzan una precipitación promedio anual de 1016 a $1132.2 \mathrm{~mm}$; la temperatura promedio anual es de $26.2{ }^{\circ} \mathrm{C}$, con valores mínimos de $17^{\circ} \mathrm{C}$ en invierno y máximos de $36^{\circ} \mathrm{C}$ en verano (Flores y Espejel, 1994; García, 1988). b) La vegetación sobresaliente en el sur de Campeche es 
la selva mediana subperennifolia, con un dosel promedio a los $25 \mathrm{~m}$ de altura; además, existen la selva mediana caducifolia, selva alta, selva baja inundable y vegetación modificada (Flores y Espejel 1994; INEGI, 2001; March, 1994; Miranda, 1958). El clima que predomina en esta zona es cálido y subhúmedo, con lluvias de junio a octubre y con una precipitación promedio anual de $1290 \mathrm{~mm}$ (García, 1988; March, 1994). La temperatura varía de 4 a $44{ }^{\circ} \mathrm{C}$, con un promedio de $26{ }^{\circ} \mathrm{C}$ (CIECAS-IPN y SEMARNAP-Campeche, 1998).

Chrotopterus auritus (Peters, 1856)

El 22 de febrero de 2001 se capturó un individuo macho (122-10-21-45, AB $=81$, masa corporal, $\mathrm{MC}=76 \mathrm{gr})$ con redes a ras de suelo en Xbonil $\left(18^{\circ} 30^{\prime} 38.3^{\prime \prime} \mathrm{N}\right.$, $90^{\circ} 02^{\prime} 47.7^{\prime}$ ' W), en un selva mediana conservada del municipio de Calakmul. El espécimen está depositado en la colección de mamíferos del Museo de Zoología de ECOSUR Chetumal (ECO CHM) con número de catálogo 2037. Esta especie ha sido reportada al norte de los estados de Yucatán y Quintana Roo (Hall, 1981) y en Campeche (López-Wilchis y López-Jardinez, 1998). Sin embargo, los últimos autores no indican la localidad de registro. La especie es considerada rara, pero ampliamente distribuida (Reid, 1997). Nuestro espécimen constituye el segundo registro para Campeche y confirma la distribución intermedia entre los registros de Chiapas y el norte de la Península de Yucatán.

Vampyrum spectrum (Linnaeus, 1758)

El 23 de febrero de 2001 se capturó a una hembra (138.6-0-29.4-35.6, $\mathrm{AB}=$ $104, \mathrm{MC}=113 \mathrm{gr}$; ECO CHM 2033) y un macho (133.8-0-31.2-39.8, AB = 108.6, $\mathrm{MC}=112$ gr; ECO CHM 2034) y el 19 de julio de 2001 se capturó otro macho con testículos escrotados (145-0-27-44-15, $\mathrm{AB}=105.8, \mathrm{MC}=175 \mathrm{~g}$; Colección Osteológica de Comparación del Laboratorio de Arqueozoología "M. en C. Ticul Álvarez Solórzano" del Instituto Nacional de Antropología e Historia) con redes a ras de suelo en Xbonil $\left(18^{\circ} 30^{\prime} 38.3^{\prime \prime} \mathrm{N}, 90^{\circ} 02^{\prime} 47.7^{\prime}, \mathrm{W}\right)$, en una selva mediana conservada del municipio de Calakmul. Esta especie fue reportada anteriormente por Hernández-Huerta et al. (2000) para la Península de Yucatán a $76.3 \mathrm{~km}$ SE de nuestro sitio de de captura en el sur de Campeche. La especie es considerada rara y local (Reid, 1997). Nuestros especímenes constituyen los segundos registros para el Estado y la Península de Yucatán.

\section{Molossus rufus É. Geoffroy St.- Hilaire, 1805}

El 6 de julio de 2000 se encontró un ejemplar macho subadulto muerto (13545-10-16, AB = 49.2; ECO CHM 2068) en el centro de la ciudad de Campeche $\left(19^{\circ}\right.$ 50 ' 41.6' N, $90^{\circ} 32$ ' 20.8' 'W). El 22 de octubre de 2000 colectamos dos hembras adultas $(130-43-10-19, \mathrm{AB}=50, \mathrm{MC}=35 \mathrm{~g}, \mathrm{ECO} \mathrm{CHM} 2044 ; 120-38-10-18, \mathrm{AB}=$ 48.3, MC $=33$ g, ECO CHM 2045) y, el 4 y 5 de mayo de 2001 se capturó y liberó una hembra $(\mathrm{AB}=53.6, \mathrm{MC}=48 \mathrm{~g})$ y un macho $(\mathrm{AB}=43, \mathrm{MC}=45.8 \mathrm{~g})$ en el ejido Nayarit de Castellot, Champotón, Campeche (19 $\left.13^{\circ} 24.7^{\prime \prime} \mathrm{N}, 90^{\circ} 14^{\prime} 55.5^{\prime \prime} \mathrm{W}\right)$. El 
tipo de vegetación del lugar corresponde a un fragmento de selva mediana modificada, donde predominan árboles de 25 a $30 \mathrm{~m}$ de altura. El 20 de diciembre de 2001 capuramos y liberamos a una hembra $(\mathrm{AB}=51.3, \mathrm{MC}=39 \mathrm{~g})$ y un macho $(\mathrm{AB}=50$, $\mathrm{MC}=45 \mathrm{~g})$ adultos en la zona arqueológica de Nadzcaan $\left(18^{\circ} 32^{\prime} \mathrm{N}, 89^{\circ} 55^{\prime} \mathrm{W}\right)$. La vegetación de este último lugar se caracteriza por una selva mediana subcaducifolia. El primer registro de esta especie para Campeche fue dado a conocer por HernándezHuerta et al. (2000) a $72.7 \mathrm{~km} \mathrm{SE}, 157 \mathrm{~km} \mathrm{SE} \mathrm{y} 232 \mathrm{~km}$ SE de nuestros sitios de captura. Nuestros especímenes constituyen el segundo registro de $M$. rufus para el Estado. La especie es considera con distribución amplia para México (Hall, 1981), pero se desconoce aspectos biológicos y ecológicos dentro de la región.

\section{Galictis vittata Nelson, 1901}

El 1 de mayo de 2002 encontramos por la tarde un grisón macho (730-182$78-32, \mathrm{MC}=2.5 \mathrm{~kg}$; ECO CHM 2067) con testículos escrotados atropellado en la carretera Campeche - Mérida a $39 \mathrm{~km} \mathrm{~N}$ de la ciudad de Campeche (19 56' 54.06"' $\mathrm{N}, 90^{\circ} 21^{\prime} 48.38^{\prime}$ ' W). El hábitat circundante al sitio de colecta corresponde a una selva mediana con un ambiente modificado cercano a la zona costera de Campeche (INEGI, 2001). Reid (1997) y G. Ceballos (com. pers.) han mencionado que se les encuentra en zonas bajas y cuerpos de agua. Su historia natural es poco conocida. Su presencia ha sido reportada al norte de la Península de Yucatán (Hall, 1981; Hernández Betancourt et al., 1996; Navarro et al., 1990; Wilson, 1991) y al sur de Quintana Roo (Pozo de la Tijera et al., 1991). Con nuestro registro, además de ser el primero para el Estado, se amplia el área de distribución de la especie a $226.5 \mathrm{~km}$ al NW del registro más cercano (Pozo de la Tijera et al., 1991) e incrementa el número de carnívoros de 13 a 14.

\section{Cueva "Volcán de los Murciélagos"}

La cueva se localiza en la ampliación forestal de Hopelchén ( $18^{\circ} 31^{\prime} 22.15^{\prime}$ " $\mathrm{N}, 89^{\circ} 49^{\prime} 25.07^{\prime}$ ' W) en el municipio de Calakmul. La vegetación circundante a la cueva corresponde a una mezcla de selva mediana y vegetación secundaria. La información sobre esta cueva es casi nula y regionalmente es una de las de mayor tamaño (Reddell, 1981). Se realizó una visita el 29 de abril de 2001 y con el uso de una red de mano se registraron siete especies de murciélagos insectívoros (Mormoops megalophylla, Pteronotus parnellii, P. davyi, P. personatus, Natalus stramineus, Myotis keaysi y Nyctinomops laticaudatus). El 2 de mayo de 2002 se visitó la cueva y mediante el uso de una videocámara se grabó la salida de los quirópteros desde las 18:30, antes del anochecer hasta que ya no se registró salida de murciélagos, con la cual se hizó una estimación de la población que va entre 80,000 a 100,000 murciélagos. Además se observó depredación de murciélagos por aves como Sturina nitida, Tyto alba, Ciccaba nigrolineata, Cyanocorax morio y C. yucatanicus. Por otra parte, empleando la clasificación del refugio basada en la diversidad y abundancia de murciélagos de Arita (1993), esta cueva puede ser considerado como un refugio multiespecífico con alta abundancia. En términos ecológicos esta cueva debería ser 
considerada como un elemento prioritario para la conservación a nivel regional.

Los autores en los últimos años han recibo diversos apoyos para realizar diferentes investigaciones, como del Consejo Nacional de Ciencia y Tecnología (No. 60371), Fundación Pablo García, The Natural Conservancy - PRONATURA Península de Yucatán y Secretaría de Ecología del Gobierno del Estado de Campeche. Además, agradecemos a H. Zarza, J. Pacheco y G. Ceballos por sus valiosos comentarios en la preparación del manuscrito. A las autoridades de la UMA de Xbonil por permitirnos utilizar su infraestructura. Asimismo, a J. Morales, J. M. Weber Rodríguez, M. Miranda, G. Reyes, A. López, D. Simá, L. Dzul y K. Arcique por el apoyo logístico brindado en el campo.

\section{LITERATURA CITADA}

Arita, H. T. 1993. Conservation biology of the cave bats of México. Journal of Mammalogy, 74:693-702.

CIECAS-IPN y SEMARNAP. 1998. Programa de desarrollo regional sustentable. Diagnóstico comunitario. Calakmul, Campeche. CIECAS-IPN y SEMARNAP Campeche, Campeche.

Flores, J. S. e I. Espejel-Carvajal. 1994. Tipos de vegetación de la Península de Yucatán. Universidad Autónoma de Yucatán. Etnoflora Yucatanense, Fascísculo 3.

García, E. 1988. Modificaciones al sistema de clasificación climática de Köppen. Instituto de Geografía. Universidad Nacional Autónoma de México. México, D.F.

Genoways, H. H. y J. K. Jones Jr. 1975. Annotated checklist of mammals of the Yucatán Peninsula, Mexico. IV. Carnivora, Sirenia, Perissodactyla, Artiodactyla. Occasional Papers of the Museum, Texas Tech University, 26:1-22.

Hall, E.R. 1981. The Mammals of North America. John Wiley \& Sons. 2nd. ed. E.U.A.

Hernández Betancourt, S., V. Sánchez C-Cordero, J. Sosa Escalante y A. Segovia Castillo. 1996. VIII. Lista anotada de los mamíferos terrestres de la Reserva de Dzilám, Yucatán, México. Listados faunísticos de México. No. 8. Instituto de Biología, Universidad Nacional Autónoma de México, México, D. F.

Hernández-Huerta, A., V. J. Sosa, J. M. Aranda y J. Bello. 2000. Noteworthy records of small mammals from the Calakmul biosphere reserve in the Yucatán Peninsula, Mexico. The Southwestern Naturalist, 45:340-344.

INEGI. 2001. Inventario Nacional Forestal 2000-2001. Instituto Nacional, Estadística, Geografía e Informática. México, D.F.

Jones, J. K. Jr., J. D. Smith y H. H. Genoways. 1973. Annotated checklist of mammals of the Yucatán Peninsula, Mexico. I. Chiroptera. Occasional Papers of the Museum, Texas Tech University, 13:1-31.

Jones, J. K. Jr., H. H. Genoways y T. E. Lawlor. 1974a. Annotated checklist of mammals of the Yucatán Peninsula, Mexico. II. Rodentia. Occasional Papers of the Museum, Texas Tech University, 22:1-31.

Jones, J. K. Jr., H. H. Genoways y J.D. Smith. 1974b. Annotated checklist of mammals of the 
Yucatán Peninsula, Mexico. III. Marsupialia, Insectivora, Primates, Edentata, Lagomorpha. Occasional Papers of the Museum, Texas Tech University, 23:1-12.

López-Wilchis, R. y J. López-Jardinez. 1998. Los mamíferos de México depositados en coleciones de Estados Unidos y Canada. Vol. 1. Universidad Autónoma Metropolitana Unidad Iztapalapa. México, D.F.

March M., I. J. 1994. Situación actual del tapir en México. Centro de Investigaciones Ecológicas del Sureste. Serie Monográfica No. 1.

Medellín, R. A., A. L. Gardner y J. M. Aranda. 1998. The taxonomic status of the Yucatán brown brocket, Mazama pandora (Mammalia: Cervidae). Proceedings of the Biological Society of Washington, 111:1-14.

Miranda, F. 1958. Estudios acerca de la vegetación. Pp. 215-271, en: Los recursos naturales del sureste y su aprovechamiento. Ediciones del Instituto Mexicano de Recursos Naturales Renovables. México, D.F. Vol. 2.

Navarro L., D., T. Jiménez A. y J. Juárez G. 1990. Los mamíferos de Quintana Roo. Pp. 371450, en: Diversidad Biológica en la Reserva de la Biosfera de Sian Ka'an, Quintana Roo, México (D. Navarro L. Y J. G. Robinson, eds). Centro de Investigaciones de Quintana Roo y Program of Studies in Tropical Conservation, University of Florida.

Pozo de la Tijera, C., E. Escobedo Cabrera, J. L. Rangel Salazar y P. Viveros León. 1991. Fauna. Pp. 49-78, en: Estudios ecológicos preliminares de la zona sur de Quintana Roo (T. Camarena-Luhrs y S. Salazar-Vallejo, eds.). Centro de Investigaciones de Quintana Roo, Chetumal, Quintana Roo.

Reid, F. A. 1997. A field guide to the mammals of Central America and Southeast Mexico. Oxford University Press, Oxford.

Reddell, J. R. 1981. A review of the cavernicole, fauna of Mexico, Guatemala, and Belize. Texas Memorial Museum, the University of Texas at Austin. Bulletin, 27:1-327.

Sánchez H., C. y M. L. Romero. 1995. Murciélagos de Tabasco y Campeche: una propuesta para su conservación. CUADERNOS 24. Instituto de Biología, Universidad Nacional Autónoma de México.

Vargas-Contreras, J. A., J. Arroyo-Cabrales, M. Sanvicente-López y R. Reyna-Hurtado. En prensa. Nuevos registros de mamíferos para Campeche. Vertebrata Mexicana, 11.

Vargas-Contreras, J. A., J. R. Herrera-Herrera y J. E. Escobedo-Cabrera. En revisión. Noteworthy records of mammal from Campeche, México. Sometido a Mammalia.

Wilson, D. E. 1991. Especímenes tipo de mamíferos mexicanos en el National Museum of Natural History, Washington, D. C. EUA. Pp. 287-318, en: Contribuciones mastozoológicas en Homenaje al Dr. Bernardo Villa Ramírez (F. A. Cervantes, comp.). Anales del Instituto de Biología, Universidad Nacional Autónoma de México. Serie Zoológica, 62:151-382. 